\title{
Bone mineral density modeling via random field: normality, stationarity, sex and age dependence
}

\author{
Petr Henyša ${ }^{a}$, Miroslav Vořechovskýb, Michal Kuchařc ${ }^{c}$ Axel Heinemann ${ }^{\mathrm{e}}$, Jiř́ Kopal $^{\mathrm{a}}$, Benjamin Ondruschka ${ }^{\mathrm{e}}$, Niels Hammer ${ }^{\mathrm{d}}$ \\ ${ }^{a}$ Institute of New Technologies and Applied Informatics, Faculty of Mechatronics, Informatics and Interdisciplinary Studies, Technical University of Liberec, \\ Studentská 1402/2, 46117 Liberec, Czech Republic \\ ${ }^{b}$ Institute of Structural Mechanics, Faculty of Civil Engineering, Brno University of Technology, Veveř́ 331/95, 60200 Brno, Czech Republic \\ ${ }^{c}$ Department of Anatomy, Faculty of Medicine in Hradec Králové, Charles University, Šimkova 870, 500 03, Hradec Králové, Czech Republic \\ ${ }^{d}$ Department of Macroscopic and Clinical Anatomy, Medical University of Graz, Auenbruggerpl. 2, 8036 Graz, Austria \\ ${ }^{e}$ Institut für Rechtsmedizin, Universitätsklinikum Hamburg-Eppendorf, Butenfeld 34, 22529 Hamburg, Germany
}

\begin{abstract}
Background and Objective: Capturing the population variability of bone properties is of paramount importance to biomedical engineering. The aim of the present paper is to describe variability and correlations in bone mineral density with a spatial random field inferred from routine computed tomography data.

Methods: Random fields were simulated by transforming pairwise uncorrelated Gaussian random variables into correlated variables through the spectral decomposition of an age-detrended correlation matrix. The validity of the random field model was demonstrated in the spatiotemporal analysis of bone mineral density. The similarity between the computed tomography samples and those generated via random fields was analyzed with the energy distance metric.

Results: The random field of bone mineral density was found to be approximately Gaussian/slightly left-skewed/strongly right-skewed at various locations. However, average bone density could be simulated well with the proposed Gaussian random field for which the energy distance, i.e., a measure that quantifies discrepancies between two distribution functions, is convergent with respect to the number of correlation eigenpairs.

Conclusions: The proposed random field model allows the enhancement of computational biomechanical models with variability in bone mineral density, which could increase the usability of the model and provides a step forward in in-silico medicine.
\end{abstract}

Keywords: shape registration, Karhunen-Loève expansion, finite element method, uncertainty quantification

\section{Introduction}

The structural and intrinsic properties of bone are inho- ${ }^{22}$ mogeneous, and vary across the multiple spatial and temporal scales and population. It has been documented that ${ }_{25}^{24}$ bone properties vary at the collagen fibrils level as well as ${ }^{25}$ the lamellae level, and naturally vary across anatomical sites ${ }_{27}^{26}$ [1]. Structural inhomogeneities are related to bone fragility ${ }_{28}$ and toughness $[2,3,4,5]$. Bone mineral density (BMD) is widely used to study bone properties. BMD is remarkably inhomogeneous $[2,6]$, and is connected to bone elasticity and ${ }_{31}^{30}$ fracture risk $[7,8,9]$.

The spatial variation of BMD has previously been analyzed ${ }^{32}$ through variograms $[10,11]$, where the authors attempted to enhance the fracture risk prediction ability related to BMD. ${ }_{35}^{34}$ Other studies have demonstrated significant correlations between the parameters of BMD variograms and both trabecu- ${ }^{36}$ lar bone morphological measures and bone strength $[12,13]{ }^{37}$ On the other hand, no significant correlation was found between vertebrae strength and variogram parameters [14]. Dong et al. [15] demonstrated that bone elasticity variation ${ }_{40}$

\footnotetext{
*Corresponding author

Email address: michal . kuchar@lfhk.cz (Michal Kuchař)
} to the remodeling process in bone, stationarity and isotrop-
icity assumptions are likely to be violated, but to the authors' knowledge, this has never been investigated. Recent studies have emerged describing bone properties as a random field. Desceliers et al. [16] introduced a simplified random field model of cortical bone, but it has not yet been calibrated using clinical data. Another study showed that trabecular structure can be generated by an inverse Monte Carlo simulation on Voronoï cells, which exhibited a good match with trabecular morphology [17]. In the study by Luque et al. [18], a density random field of a trabecular region of interest (ROI) was modeled with directionally separable autocorrelation functions based on computer tomography (CT). So far, this study by Luque et al. [18] can be considered the first and also only one that considers density as a random field. However, the conclusions in their study are difficult to generalize to the whole bone because they were derived from a bone sample of small size under stationarity conditions.

Unstable pelvic fractures are difficult to treat and current methods of fixation suffer from a high failure rate $[19,20,21]$. A higher risk of fracture fixation failure is associated with lower mineral density values $[22,23]$. In addition, 
local variance in mineral density has been shown to signif- 97 icantly affect the strength of fixation screws [24]. Regional ${ }_{98}$ variance of pelvic bone density is insufficiently described in ${ }_{99}$ the literature, although it may play an important role in the ${ }_{100}$ study of pelvic fractures. Therefore, the pelvic bone serves as ${ }_{101}$ a suitable candidate to demonstrate BMD as a random field ${ }_{102}$ in the present study.

\section{Study Aim \& Outline}

The present study aims to analyze the spatio-temporal vari-10 ability in BMD of the pelvic bone and to model BMD as a ran-107 dom field. First, a shape registration algorithm was used to ${ }^{108}$ geometrically align CT samples (Shape Registration section). ${ }^{109}$ In the next step, the Karhunen-Loève expansion (KLE) was'110 employed to simulate BMD as a random field with Gaussian ${ }^{111}$ coefficients, see the Karhunen-Loève Expansion section. The ${ }^{112}$ new BMD realizations based on the random field model were ${ }^{113}$ validated using average bone mineral density (BMD), which ${ }^{114}$ can be considered a global measure of bone mineral density. ${ }^{115}$ Furthermore, what is known as the energy distance [25] was ${ }^{116}$ computed between the random field of BMD obtained from ${ }^{117}$ the CT samples and those generated with the KLE. The en-118 ergy distance is evaluated locally to see how similar the distri-119 butions are point-wise, and then also globally as an integral120 measure (Validation Measures section); see the flow chart in ${ }^{121}$ Figure 1.

\section{Materials and Methods}

\section{CT Data Collection}

The anonymized retrospective CT data of 97 females and 88 males were randomly taken from routine examinations performed in the Faculty Hospital in Hradec Králové under ethical approval 202102IO2P. The CT resolution of the dataset was $0.8 \times 0.8 \times 0.8 \mathrm{~mm}$ (Siemens Definition AS+, Siemens Definition 128, both Siemens AG, Erlangen, Germany; 120-130 kV using CareDose, reconstruction kernel 80-90, bone algorithm). The inclusion criteria were as follows: abdominal CT scans, bones without any trauma and, an age range of 20 years or older. Patients who had no record of having undergone a densitometric examination at the time of data collection (2018-2020) were selected. The sample population age per sex is in the range of $22-88$ years, divided into 10 bins, where each bin contains more than 5 samples. The pelvic bone geometry implicitly defined by Hounsfield (HU) field was extracted with MITK-GEM interactive segmentation software. First, the rough contours of the bone and background were drawn manually on several slices. Subsequently, the GraphCut algorithm was used to segment the rest of the slices [26].

The CT scans were calibrated internally resulting in BMD [27]. The HU values of air, bone tissue, fat, blood and muscle were considered for internal calibration as shown in Figure 2. Only the right-hand side pelvic bone was considered because no significant difference was identified between the left and right sides.

\section{Shape Registration}

The estimation of the random field density requires the universal description of bone locations among all of the experimentally studied bones using a single reference/template bone shape. This is achieved by introducing a fixed metric for spatial or temporal locations per sample to evaluate at. This requirement is violated for bone samples because each sample has a different size and shape. However, bone samples are anatomically and topologically equivalent. This implies the existence of a point correspondence between two shapes under some suitable class of bijective maps and similarity metrics. To find such a correspondence, rigid and affine transforms were realized for the initial global alignment of bones in datasets using the ANTs registration library [28]. Mutual Information (MI) was used as a similarity metric [29]. Then, a non-linear transform was found with the help of the SyN diffeomorphic-based registration algorithm in the ANTs library, see [28, 29]. The similarity of deformed bone shapes was measured with a modified intensity-based criterion called the demons-like metric. This metric provides the best accuracy/speed balance out of all the metrics tested (mean-squared difference, cross-correlation, MI) [29, 28]. In order to minimize registration error, a template bone shape, which is an estimate of the mean sample shape, was estimated according to [29, 30]. The computer analysis of BMD in the original CT data space is inefficient. Therefore, the BMD is projected into a suitable space with fewer DOFs. In fact, this projection is an approximation of the BMD by piecewise (dis)continuous functions using the least squares method. This approach leads to the minimization of the following functional:

$$
\Pi:=\int_{\Omega}(\mathrm{R}(\hat{\rho}, \bar{\rho}))^{2} \mathrm{~d} \Omega
$$

The goal is to find an approximation of the BMD that best represents the original CT data. The residual $\mathrm{R}$ represents the difference between the CT BMD value $\bar{\rho}$ and the approximated value with unknown coefficients $\hat{\rho}$ :

$$
\mathrm{R}(\hat{\rho}, \bar{\rho}):=\phi \hat{\rho}-\bar{\rho}
$$

The $\phi$ is FE basis functions evaluated at a given integration point. Substituting (2) into (1) and taking the derivative with respect to coefficients $\hat{\rho}$, one gets:

$$
\frac{\partial \Pi}{\partial \hat{\rho}}=\int_{\Omega} 2 \phi \phi^{T} \hat{\rho}-2 \phi \bar{\rho} \mathrm{d} \Omega=0 .
$$

This expression represents a system of linear equations for unknown values of $\hat{\rho}$ :

$$
\mathbf{K} \hat{\boldsymbol{\rho}}=\mathbf{f}
$$




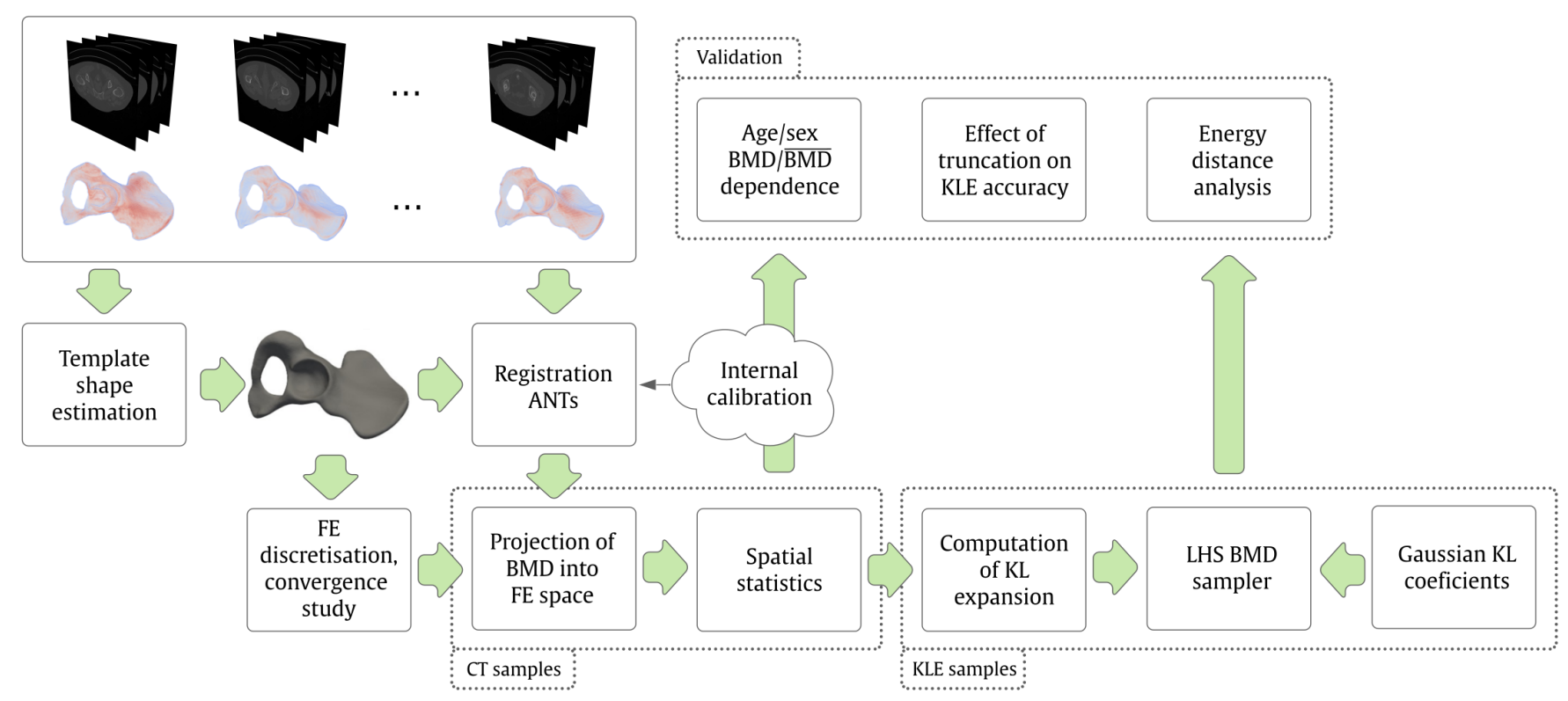

Figure 1: A flowchart of the study.

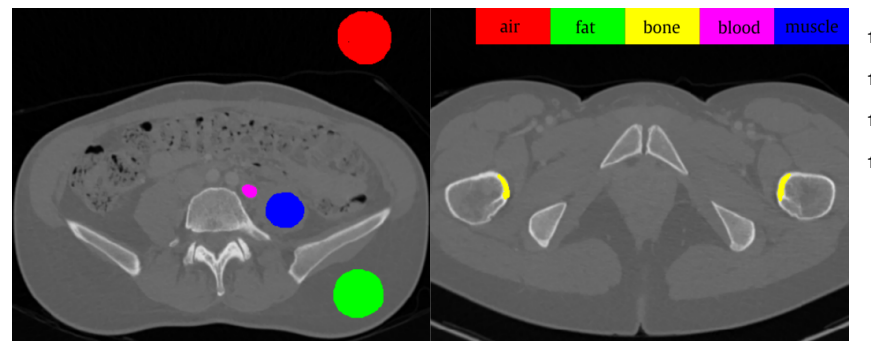

Figure 2: Example of CT slice where $\mathrm{HU}$ values of the considered tissues were selected for internal calibration. ROI content $\left(\mathrm{mm}^{2}\right)$ : air: 1312 ; fat: 1109; bone: 160; blood: 92; muscle: 618. Mean HU (standard deviation): air: -1002(7); fat: -90(12); bone: 1233(236); blood: 217(16); muscle: 60(12).

where $\mathbf{K}=\int_{\Omega} \phi \phi^{T} \mathrm{~d} \Omega$ and $\mathbf{f}=\int_{\Omega} \phi \bar{\rho} \mathrm{d} \Omega$. Note that $\bar{\rho}$ can be noisy, and hence it is evaluated by averaging in a sampling volume of $4 \times 4 \times 4$ voxels in size.

There are two sets of finite element (FE) models used in the present study. The first set consists of validation models. The morphed BMD fields from the dataset were projected onto a discontinuous FE space constructed on the template mesh. All samples in the dataset shared the same geometry domain and finite element space. The correlation matrix of BMD can then be estimated. The FE models in the second set contain BMD fields simulated by KLE on the template geometry. The FE mesh size was estimated based on an auxiliary convergence study where a $\overline{\mathrm{BMD}}$ difference between two mesh refinements of below $5 \%$ was considered to be converged. The resultant number of degrees of freedom (DOFs) was roughly $M \approx 0.7 \cdot 10^{6}$.

\section{Karhunen-Loève Expansion}

The data set was split into two sets according to sex in order to capture sex differences. Consequently, the relation
146 between age and BMD was analyzed and linear regression was used to separate deterministic trends composing of intercept (sample mean) $\boldsymbol{\rho}_{0}$ and slope $\boldsymbol{\rho}_{1}$ from the data matrix $\mathrm{X}$.

The random field $\rho(\mathrm{x}) \in \Omega$ is not known explicitly, but only through a set of $N$ standardized realizations projected onto the template bone:

$$
\mathbf{X}=\left\{\mathbf{X}_{1}, \mathbf{X}_{2}, \ldots, \mathbf{X}_{M}\right\}, \mathbf{X} \in \mathbb{R}^{M, N}
$$

The projected realizations are evaluated at DOF coordinates, from which the matrix of realizations $\mathbf{X}$ is built. The empirical correlation matrix $\mathbf{C}$ is estimated as $\frac{1}{N-1} \mathbf{X X}^{T}$. The discretized random field can be viewed as a set of correlated random variables. Sample paths of Gaussian random fields can then be generated by transforming uncorrelated Gaussian random variables into correlated space [33, 34]. One possible linear mapping between the uncorrelated and correlated Gaussian random vectors is via the $\mathrm{KL}$ expansion. This expansion involves the eigen-decomposition of the correlation matrix (or the covariance function having the role of a covariance kernel in the continuous version of the $\mathrm{KL}$ expansion). In order to compute the $\mathrm{KL}$ decomposition of $\mathbf{C}$, the associated discrete eigenvalue problem must be solved [35]:

$$
\mathrm{C} \Psi=\mathrm{D} \Psi
$$

where $\Psi \in \mathbb{R}^{M, M}$ is a matrix of eigenvectors and $\mathbf{D}=$ $\operatorname{diag}\left(\lambda_{1}, \lambda_{2}, \ldots, \lambda_{M}\right)$ is the diagonal matrix of eigenvalues. The full population of correlation matrix $\mathrm{C}$ is impossible as it is dense, moreover the rank of the matrix $\mathrm{C}$ is $N$ only and hence we adopt an alternative solution to the above eigenproblem represented by a suitable matrix decomposition. Considering an economical QR decomposition of $\mathbf{X}$, the matrix $\mathbf{C}$ can be expressed:

$$
\mathbf{C}=\mathbf{Q R R}^{T} \mathbf{Q}^{T}, \mathbf{R R}^{T} \in \mathbb{R}^{N, N} .
$$


Consequently, the singular value decomposition of product ${ }_{191}$ $\mathbf{R R}^{T}$ is computed:

$$
\mathbf{R R}^{T}=\mathbf{V D V}^{T}
$$

Substitution of Eq. (8) into Eq. (7) leads to:

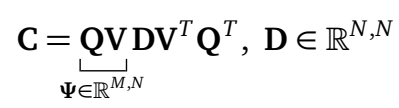

where $\boldsymbol{\Psi}$ and D are the eigenvector and eigenvalue matri- ${ }^{198}$ ces of $\mathbf{C}$. Once the $N$ eigenpairs have been computed and ${ }^{199}$ sorted in decreasing order $\lambda_{1} \geq \lambda_{2}, \ldots, \lambda_{N-1} \geq \lambda_{N}$, the spec- ${ }^{200}$ tral representation of random field $\boldsymbol{\rho}(\mathbf{x})$ can be replaced with ${ }^{201}$ a truncated discrete $\mathrm{KL}$ expansion [35]:

$$
\boldsymbol{\rho}(\mathbf{x})=\boldsymbol{\rho}_{0}(\mathbf{x})+\boldsymbol{\rho}_{1}(\mathbf{x}) t+\boldsymbol{\sigma}(\mathbf{x}) \sum_{i=1}^{P} \sqrt{\lambda_{i}} \theta_{i} \psi_{i}(\mathbf{x})
$$

where $\theta_{i}$ is a zero mean, unit variance $i$ th Gaussian pairwise $e_{206}$ uncorrelated variable described by $\mathscr{N}(0,1), t$ is a time (age) in a range from 22-89 years (from CT data sets) and $\boldsymbol{\sigma}(\mathbf{x})$ is the sample standard deviation.

The truncation in the KLE expressed in Eq. (10) may lead to dramatic computation time savings, since $P$ can be considerably less than the order of the correlation matrix ( $=$ the number of discretization points), $M$, and also less than the $\operatorname{order} N$. An appropriate selection of truncation order $P$ can be based on various points of view. The standard way is to control the truncation error in KLE using the decay of the covariance operator's eigenvalues. The eigenvalues play the role of variances of the underlying uncorrelated random variables $\theta_{i}$, which serve as random coefficients of deterministic eigenfunctions/vectors $\boldsymbol{\psi}_{i}(\mathbf{x})$. Given this interpretation, one can easily control the total amount of variance represented via the truncated KLE. Since the correlation matrix $\mathbf{C}$ is positive (semi)definite by definition, the eigenvalues are nonnegative and their sum is known in advance. Therefore, the eigenvalues can be sorted from the maximum eigenvalue to the minimum one, along with the corresponding eigenvectors (or eigenfunctions). The gradual sum of the sorted eigenvalues serves as an indicator of how much variance is captured by the corresponding subset of eigenmodes. In other ${ }^{207}$ words, the expansion can be truncated after taking a subset ${ }^{208}$ of $P$ dominant eigenvalues (=variables with the largest vari-209 ance). The number of modes needed to cover a sufficient ${ }^{210}$ variability depends on the reach of the autocorrelation func-211 tion: when the autocorrelation length is high compared to $\mathrm{t}^{212}$ the domain dimensions, usually only a small subset of eigenpairs is necessary for a given truncation error. Furthermore, it can be shown that the $\mathrm{KL}$ expansion is optimal with respect to the global mean-squared error among all series expansions ${ }_{214}$ of truncation order $P$. We remark that, in order to achieve ${ }_{215}$ convergence, there are restrictions regarding the mesh dis- ${ }_{216}$ cretization [33].

The amount of variance captured by the truncated $\mathrm{KLE}_{218}$ may not be the only criterion for the selection of truncation ${ }_{219}$ order, $P$. We also consider stabilization of the energy distance between the generated samples and the required value with $P$ as shown in the numerical results below.
In order to generate sample paths of random fields via the $\mathrm{KL}$ expansion, a technique for the generation of the underlying standardized pairwise uncorrelated Gaussian random variables $\theta_{i}$ must be employed. Sample paths of random fields generated via orthogonal series expansion directly inherit the quality of sample statistics of the underlying random variables. As shown in [33], utilization of the stratification technique called Latin Hypercube Sampling (LHS) [36, 37] leads to faster convergence of the sample statistics to the target values with increasing number of samples than crude Monte Carlo sampling. Therefore, LHS was used to generate KLE realizations $\left(n_{\text {sim }}=300\right.$ samples were found sufficient to obtain a converged mean and standard deviation). The LHS generator of pelvic BMD realizations accompanying this paper is freely available on the BoneGen website [38].

\section{Validation Measures}

$\overline{\mathrm{BMD}}$ and energy distance [25] were considered as validation measures for the proposed BMD random field model. The $\overline{\mathrm{BMD}}$ measure is an integral value, defined as

$$
\overline{\mathrm{BMD}}=\int_{\Omega} \rho \mathrm{d} \Omega
$$

This integral is computed by finite element (FE) discretization. The $\overline{B M D}$ can be considered as the spatial average of the BMD. Since the volume is identical for all samples, it is unnecessary to include a volume denominator in expression (11). Therefore, the $\overline{\mathrm{BMD}}$ could also be interpreted as a fraction of the bone mass which is formed by mineral content. The energy distance $d$ provides a way to measure the similarity between two probability distributions. For two onedimensional distributions, $u$ and $v$, the distance $d$ is computed [25]:

$$
d(u, v)=\sqrt{2 \int_{-\infty}^{+\infty}(U(x)-V(x))^{2} \mathrm{~d} x}
$$

where $U$ and $V$ are cumulative distribution functions. ${ }^{1}$ Within this study, the expression above describes the spatial distance density over the bone volume, and hence we additionally introduce a global distance measure as well: $D=\int_{\Omega} d \mathrm{~d} \Omega$. This spatial integral over bone volume is again computed with the help of FE discretization.

\section{Results}

The mean and standard deviation functions of BMD varied spatially significantly and differed for the cortical and trabecular regions and for both females and males, i.e., BMD random fields were non-stationary in space.

Data analysis for females yielded the highest sample mean value of 1.246 (arcuate line, upper third), while the lowest

\footnotetext{
${ }^{1}$ For empirical distribution functions, the integral is replaced by a sum.
} 
was 0.106 (above the greater sciatic notch). The highest sample standard deviation (std) was 0.191 (top of the acetabular margin) while the lowest was 0.015 (deep to the auricular surface). The BMD normality is considered to be acceptable at the significance level $p \geq 0.05$, which was fulfilled for $59 \%$ of the bone volume. The skewness range is -1.893 (midpart of the anterior margin of the greater sciatic notch) to 7.502 (posterior part of the iliac wing). The negative values corresponding to left-skewed distributions occupy $23 \%$ of the volume, while the right-skewed distributions occupy $77 \%$ of the volume.

The data analysis for males yields the lowest mean value of 273 0.119 (deep to the auricular surface), while the highest was274 1.135 (uppermost part of the arcuate line). The lowest std275 was 0.016 (in between the iliac wing and the iliac tuberosity),276 while the highest was 0.218 (top of the acetabular margin).277 BMD distributions can be considered normal for $54 \%$ of the 278 volume, while the rest contained non-normally distributed 279 data. The skewness range is from -1.895 (inferior to therso ischial spine) to 6.177 (deep to the auricular surface). The281 left skewed distributions occupy $17 \%$ of the volume, whileer2 the rest of the volume was occupied by right skewed distri-283 butions. The spatial descriptive statistics are shown in Figure284 3.

Influence of KLE truncation on the accuracy of random field representation of $B M D$

The BMD was computed from CT samples and the new ${ }^{28}$ samples generated by the KLE with different numbers of eigenpairs. It was found that the most significant eigenvalue ${ }^{290}$ explains $32 \% / 36 \%$ of the variance in the BMD, and the top five explain $54 \%$ of the variance for both females and males. There is no significant statistical difference between the $\overline{\mathrm{BMD}}^{293}$ computed from CT- and KLE-based realizations, even with ${ }^{294}$ the KLE containing only the most significant eigenpair, see ${ }^{299}$ Figure 4.

\section{Age dependence of $B M D / \overline{B M D}$}

The BMD slope for females varied in range from $-5.163_{301}$ (dorsally to the arcuate line) to 3.269 (above the greater sci-302 atic notch) and from -5.470 (superior-posterior part of the acetabular margin) to 3.625 (anterior third of the iliac crest) ${ }_{304}$ $[\mathrm{mg} / \mathrm{cc} /$ year] for females and males. The BMD is interme-305 diately correlated with age at $\left(R^{2} \leq 0.51\right)$ and $\left(R^{2} \leq 0.49\right)_{306}$ for females and males, respectively. The age correlation was 307 significant at $73 \%$ and $56 \%$ of volume at a significance level 308 of $p \leq 0.05$ for females and males respectively, see Figure 5.309 At $71 \% / 61 \%$ of volume, BMD decreased with age for both ${ }_{310}$ females and males. The difference in the $\overline{B M D}$ age rate esti-311 mated from CT and KLE realizations is $5.57 \%$ and $4.71 \%$ for $_{312}$ females and males, respectively. The difference in standard ${ }_{313}$ error was $47 \%$ and $55 \%$ for females and males. The differ-314 ence in $R^{2}$ is $21 \%$ and $50 \%$ for females and males; see Table 315 1.
Table 1: Age dependence of $\overline{\mathrm{BMD}}$ estimated by linear regression for both CT and KLE samples. The KLE samples were generated with five eigenpairs included and LHS design.

\begin{tabular}{l|cc|cc}
\hline \multirow{2}{*}{ source: } & \multicolumn{2}{|c|}{ females } & \multicolumn{2}{c}{ males } \\
& CT & KLE & CT & KLE \\
\hline$\overline{\text { BMD rate }[\mathrm{mg} / \text { year }]}$ & -0.2369 & -0.2501 & -0.1168 & -0.1223 \\
standard error & 0.060 & 0.032 & 0.075 & 0.034 \\
$R^{2}$ & 0.140 & 0.169 & 0.028 & 0.042 \\
\hline
\end{tabular}

\section{Energy Distance}

The minimum/maximum distance $d_{\min } / d_{\max }$ stabilized after including more than 30 eigenpairs for females. The total distance $D$ decreased as the number of included KL pairs increased, and ended up at a value of 7425 for females.

The minimum/maximum distance $d_{\min } / d_{\max }$ decreased up to the $50^{\text {th }} \mathrm{KL}$ pair, and consequently stabilized up to the last $\mathrm{KL}$ pair. The total distance decreased as the number of included eigenpairs increased up to a minimum value of 8303 . The detailed evolution of energy distance is shown in Figure 6, together with snapshots of selected included eigenpairs. Considering only the first $\mathrm{KL}$ pair, there are energy distance peaks at the dorsal portion of the acetabular notch for females and below the anterior inferior iliac spine for males.

\section{Discussion}

Quantifying the uncertainties in bone mechanical properties originating from a representative population is of paramount importance in order to achieve clinically relevant conclusions and research-informed practice in bone treatment. Due to the complexities of bone shape and the broad individual variations materials, any biomechanical experiments, both real and virtual (for example finite element simulations), should be performed with sufficient sample size. This requirement is often difficult to achieve, and a lack of samples may reduce the potential for research conclusions to be applied to a broad population. We here introduce a random field model for BMD. With this model at hand, one can generate a number of BMD samples respecting population variability and age dependence. The current model allows the replication of the BMD density in a domain, which is a sample mean population bone shape. This step, which aims to separate BMD and shape, allows the analysis of BMD variations at a fixed metric as a random field, but it limits the model's usability. Nevertheless, shape variations can be considered as a random field as well. The study of BMD and shape variations as random fields, potentially crosscorrelated, will form the objective of subsequent studies.

Representing BMD and bone shape using random fields can be considered as a step towards creating a digital twin of bone $[39,40]$. However, the next key step is to include osteoporotic changes and analyze their effect on the random field of BMD.

Although patients without a densitometric record were selected and their CT scans were carefully examined by an 


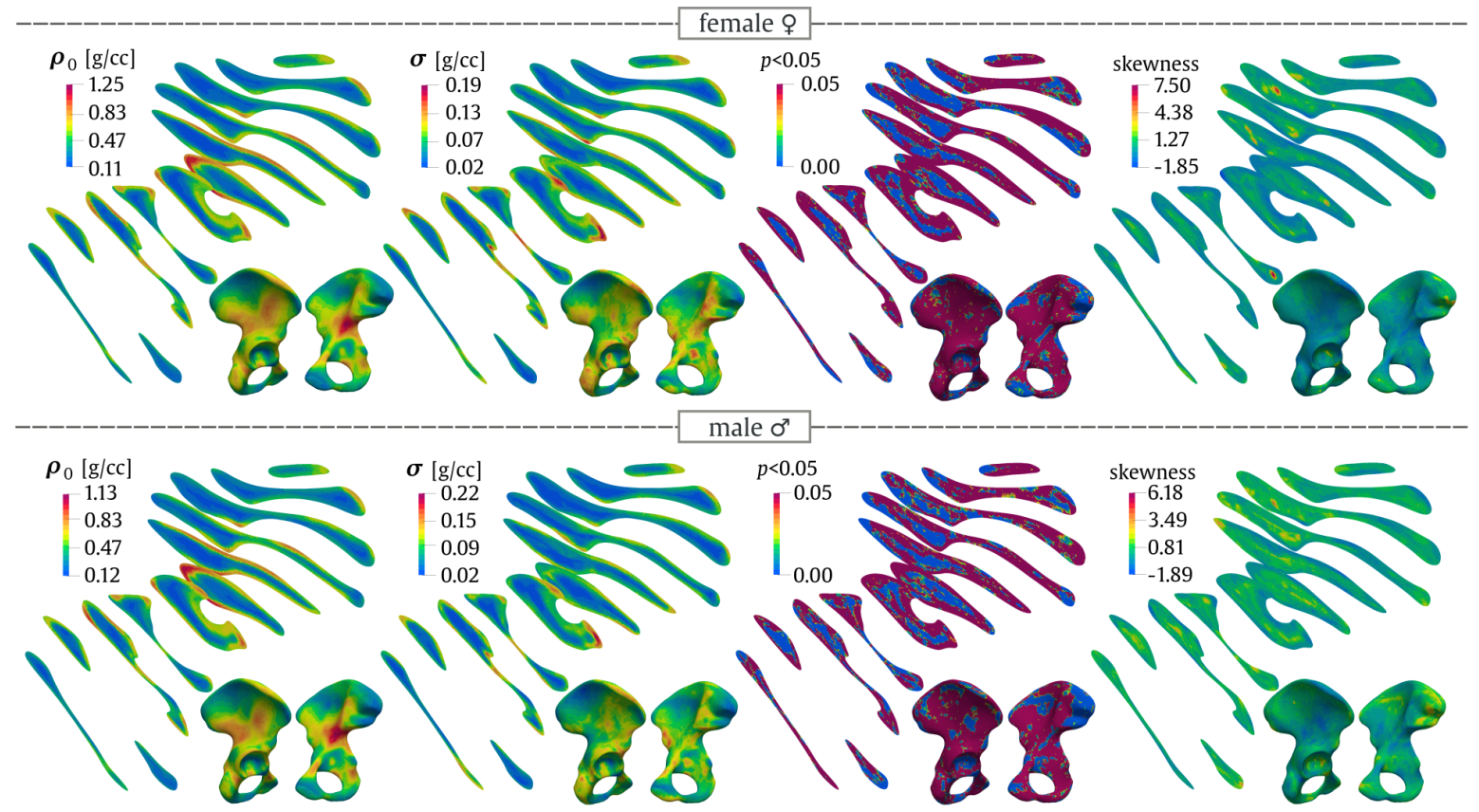

Figure 3: Spatial statistics for BMD comprising three statistical moments for both females and males

experienced radiologist, it cannot be excluded that patients 347 with osteoporosis are not present in the considered sample 348 of patients. The patients, although not listed in the database, 349 may have undergone densitometric measurements at another ${ }_{350}$ institution or may have been diagnosed with osteoporosis at ${ }_{351}$ a later date. In addition, routine CT scans provide limited in-352 formation about the patient. Furthermore, according to the $\mathrm{e}_{353}$ authors, no information is currently available in the literature 354 on the effect of internal calibration on the accuracy of the T-355 score used for osteoporosis classification. The above reasons 356 make patient selection by routine CT difficult and must be 357 considered as limitations of this study.

\section{Spatio-Temporal Dependence of a BMD Random Field}

Bone mechanical properties are well known to be age de ${ }^{361}$ pendent $([41,42,43,44])$, and it is likely that the studied ${ }^{362}$ random field will also be time dependent. For this present ${ }^{363}$ study, only the deterministic part of an age trend was iso- ${ }^{-364}$ lated. Generally, a temporal correlation structure can be ${ }^{365}$ modeled by the $\mathrm{KL}$ expansion but it requires a sufficient sam- ${ }^{-36}$ ple size per analyzed time period. Knowing the temporal ef- ${ }^{-367}$ fect on a BMD random field is extremely important and hence ${ }^{368}$ it is on the priority list for the authors' next study.

\section{Clinical CT Resolution \& Calibration}

The multi-scale nature of bone could not be considered in ${ }^{372}$ detail in the present study. The random field was estimated ${ }_{374}^{373}$ only at the organ scale based on routine CT data that may not have a sufficient resolution to capture trabecular architecture or the bone cortical shell properly. This issue complicates the ${ }^{375}$ estimation of local variations and anisotropy (fabric tensor 376 $[45,46])$ of the trabecular network as well as the composite 377 structure of the cortical shell. Although the gradient of the structure tensor might potentially be used to analyze bone anisotropy based on clinical data, this has not been tested in this study [47]. Clinical routine CT is known to distort cortical density and thickness $[48,49]$, thereby exceeding a 100\%-error in the sub millimeter structure of cortical bone. The effect of insufficient CT resolution may be seen at the central part of the iliac wing, where the thickness of the trabecular bone layers is minimized and prone to partial volume effects; this is likely to affect the random field. In some cases, even a fenestration may be present at this location [50]. It is not obvious how the statistical moments and correlation structure are affected, and a careful analysis should be performed with the help of cortical thickness and the density estimation algorithm introduced in [51], dedicated for clinical CT.

The CT data were calibrated internally, without a phantom, using surrounding tissues [27]. Recent studies have shown that internal calibration can be a full alternative to the gold phantom standard $[27,52,53]$. However, various factors that influence internal calibration remain up for debate and therefore caution is in order with regard to achieving accuracy and robustness. Fortunately, the correlation structure of the mineral density is invariant with respect to any linear calibration. However, the mean and variance of the mineral density can be biased by insufficient calibration. In an extreme case, the calibration curve can be considered a source of uncertainty in the mineral density model.

\section{Spatial Variation of BMD}

We assume that spatial fluctuation of BMD reflects the response of bones to external loading, which causes bone 

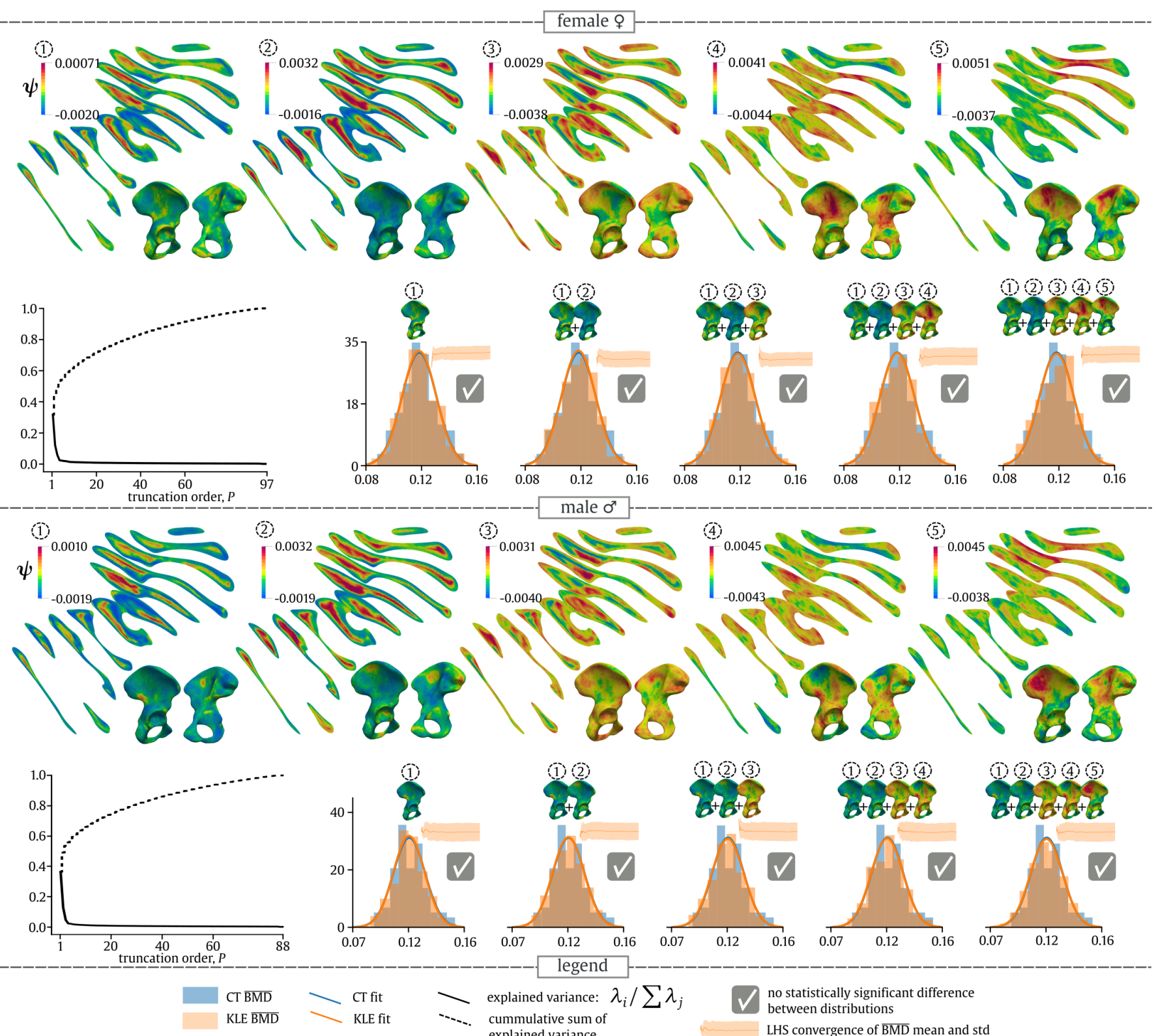

$$
\mp \begin{array}{lll}
\text { CT fit } & \cdots & \begin{array}{l}
\text { explained variance: } \lambda_{i} / \sum \lambda_{j} \\
\text { cummulative sum of } \\
\text { explained variance }
\end{array}
\end{array}
$$

no statistically significant difference between distributions

LHS convergence of $\overline{\mathrm{BMD}}$ mean and std

Figure 4: Analysis of explained variance by eigenpairs $(\lambda, \psi)$ and its influence on BMD $[\mathrm{g} / \mathrm{cc}] / \overline{\mathrm{BMD}}[\mathrm{g}]$ computed by the truncated $\mathrm{KLE}$.

to deform in a complex manner (bending + torsion + ten-393 sion/compression). The load from the trunk is directed ${ }_{394}$ through the sacroiliac (SI) joint to the acetabulum and the 395 femoral head while standing, or through the ischial tuberos-396 ity while sitting. Simultaneously, more than thirty muscles 397 and several ligaments are attached to the pelvis, loading the 398 bone with their tension in various directions. Increased $\mathrm{BMD}_{399}$ in area of the greater sciatic notch, the upper part of the arcu-400 ate line and the body of ischium seems to correspond well to 401 weight-bearing load. The relatively low standard deviation ${ }_{402}$ in this area could indicate that the weight-bearing load can 403 be considered as a common base load in the population. Even ${ }_{404}$ though the force generated by related muscles can be signif-405 icant, just slight density elevations following the margins of 406 large muscles' attachments (iliacus, gluteus medius) or iso- lated peaks for muscles with smaller insertion sites such as the rectus femoris were found. However, an interesting similarity was observed between the high standard deviations and the sites of possible apophyseal avulsions. This could indicate an increased individual localized stress induced by inserted muscles or ligament insertions (anterior superior iliac spine - rectus femoris; anterior superior iliac spine - sartorius; ischial tuberosity - hamstrings; iliac crest - abdominal wall muscles; ischial spine - sacrospinous ligament and coccygeus muscle). The increased standard deviation at these sites could reflect variations in physical activity and other unknown effects. Other sites with increased standard deviation, i.e., the superior acetabulum and anterior margin of the auricular surface, are typical of osteophytes. 
bioRxiv preprint doi: https://doi.org/10.1101/2021.02.25.432881; this version posted August 17, 2021. The copyright holder for this preprint (which was not certified by peer review) is the author/funder, who has granted bioRxiv a license to display the preprint in perpetuity. It is made available under aCC-BY-NC-ND 4.0 International license.

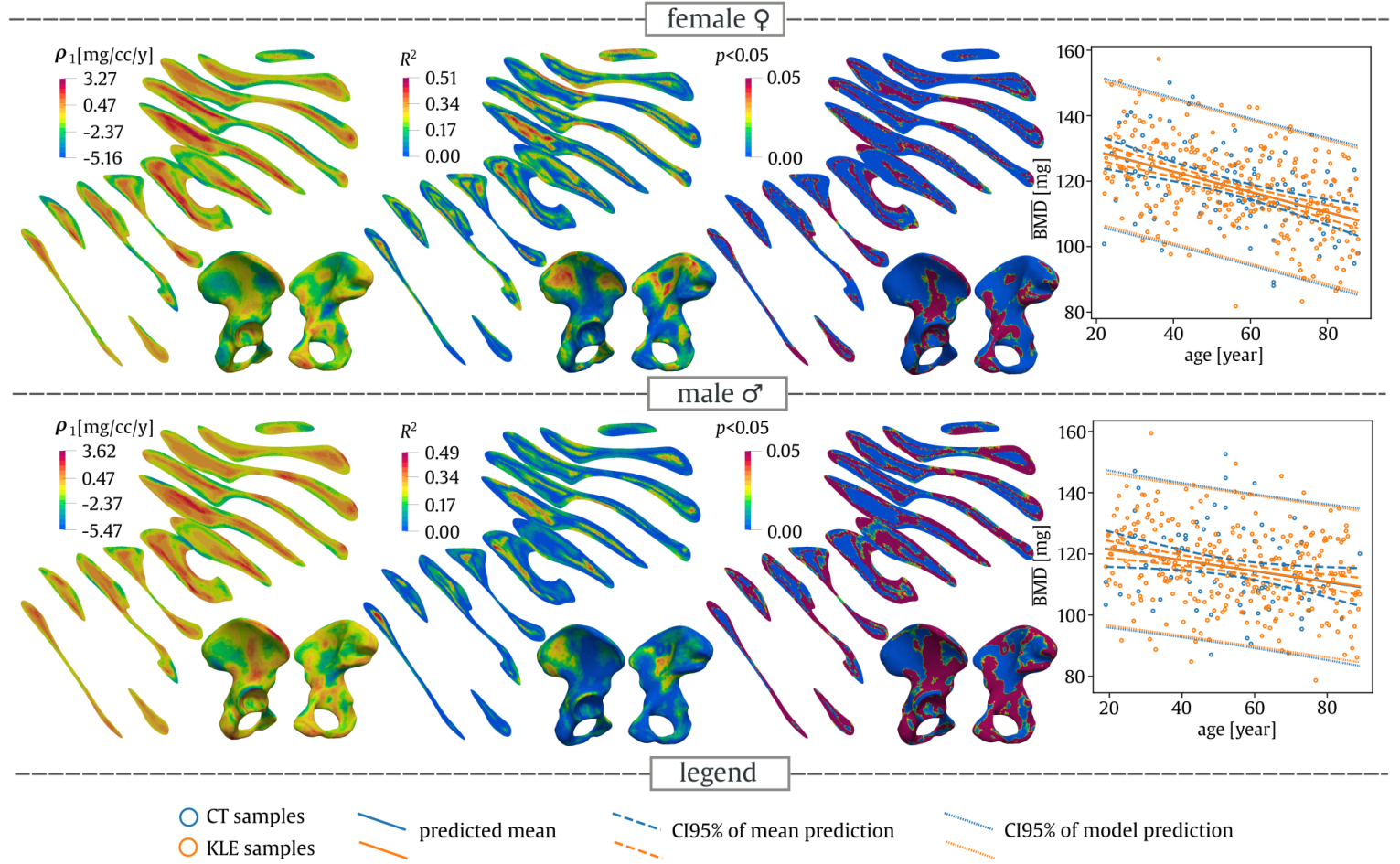

Figure 5: Spatio-temporal evolution of BMD and $\overline{\mathrm{BMD}}$.
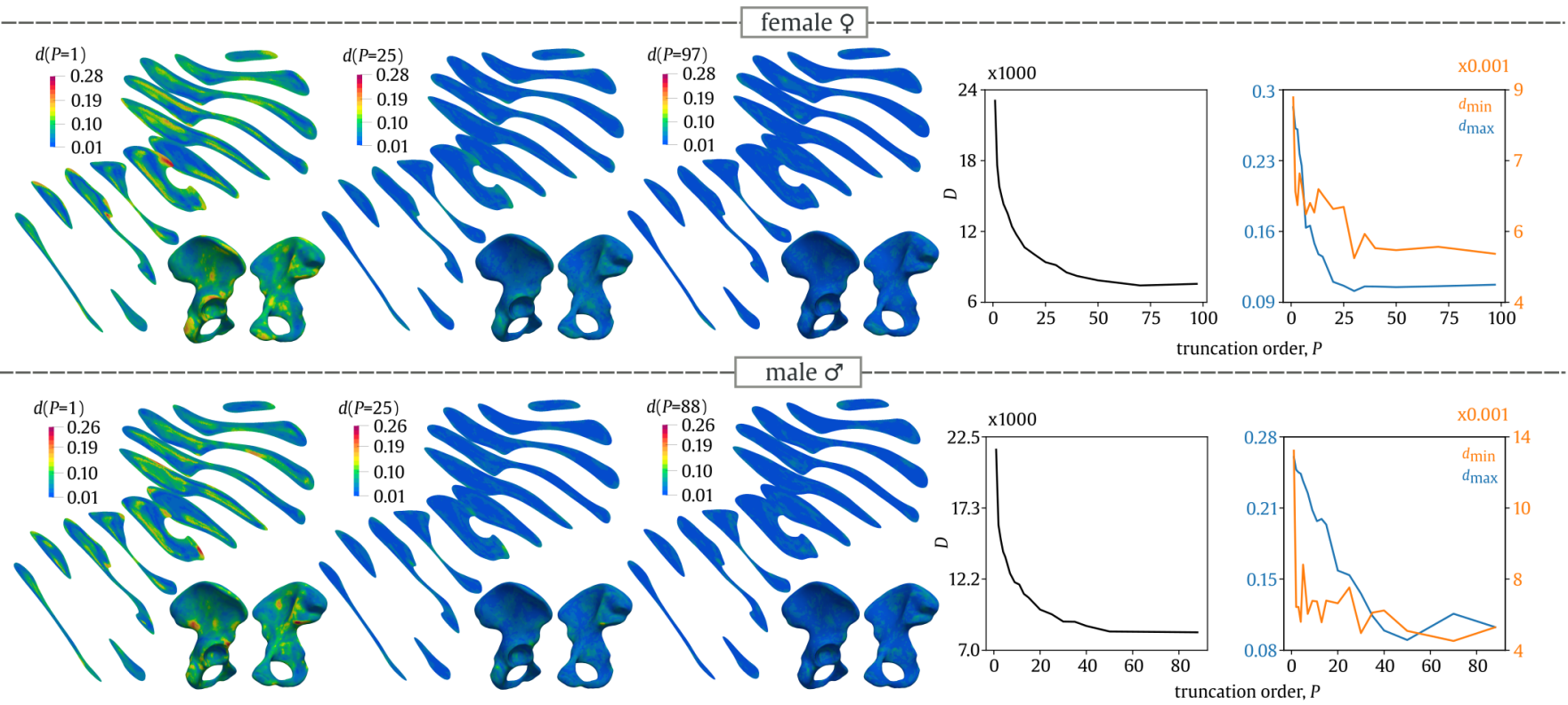

Figure 6: Spatial evaluation of the energy distance composed of spatial functions $d_{\min }$ and $d_{\max }$ and the total distance $D$ with respect to the number of eigenpairs included. The ratios $d_{\min } / d_{\max }$ are defined as the minimum/maximum distance over the domain. The minimum/maximum distance location changed with each eigenpair included, which leads to scatter in the convergence plot.

\section{Age Evolution of Bone Density}

Most publications generally assume a gradual reduction ${ }_{415}$ in bone mineral density with increasing age [54, 55, 56].416 However, it remains unclear whether this is a uniform pro- 417 cess for all skeletal sites or whether there might be some re-418 gion dependence $[57,58,59]$. Moreover, due to the variable pected $[60,61,62]$. The age changes in cortical BMD can be described by cortical thinning, higher porosity, pore diameter and osteon density [63, 64, 62, 65, 66]. Cancellous bone is affected by trabecular loss. In males this is mostly in the form 
of trabecular thinning, while in females trabecular disconnection occurs $[67,68,69,70]$. There is, however, little known about the spatial and age distribution of BMD in human innominate bone, as the majority of studies focus on long bone, vertebral or hip examinations. Our results showed general age dependent cortical BMD decline and, surprisingly, local mild trabecular BMD elevation. The reason is unclear, but it could be connected to higher trabecular mineralization patterns, which correlate with age, as documented in [71]. We found that female BMD is more sensitive to age. The BMD decreases with age in more than $68 \% / 58 \%$ of the volume of bone for females/males. The $\overline{\mathrm{BMD}}$ decreases faster for females ( $51 \%$ faster than for males).

\section{Correlation Structure of BMD}

In the present study, a non-parametric approach to the generation of new realizations of BMD has been demonstrated. This approach was based on input CT data, and the next step is to determine parametric correlation kernels, which could represent the correlation structure in time and space. It is unlikely that a simple stationary random field model for whole ${ }^{473}$ bone is achievable for several reasons: Bone forms a geo-474 metrically highly complex structure, and Euclidean distance ${ }^{475}$ is unlikely to be able to properly capture bone topology [72].476 Moreover, due to the adaptation processes that bone under-477 goes, there might be spatially dependent anisotropy in the ${ }^{478}$ correlation structure, and the distance metric will be spa- ${ }^{479}$ tially dependent. Finally, multiple latent variables coexist, for example the adaptation process, geometrical influences ${ }^{480}$ and other metabolic variables [73]. Together, these variables 481 are very likely to cause long correlation distances, as seen in ${ }_{482}$ Figure 7 . The identification and separation of these latent ${ }_{483}$ variables is difficult due to the limited information available ${ }_{484}$ from CT and from patients' medical records. This will be the ${ }_{485}$ topic of a future study. Another question concerns how well ${ }_{486}$ the empirical correlation $\mathbf{C}$ and its eigenpairs represents the ${ }_{487}$ true population correlation due to the curse of dimensionality and noise (potentially spurious correlation) [74].

\section{Assumption of Gaussian KL Coefficients}

The distribution of BMD is site dependent. There are ${ }^{491}$ locations which follow approximately normal distribution, ${ }^{492}$ while other locations are slightly left-skewed and signifi-493 cantly right-skewed in distribution as well. The proposed ${ }^{494}$ KLE-based model uses uncorrelated Gaussian coefficients, ${ }^{495}$ which introduces a certain inaccuracy that is seen in the en-496 ergy distance metric. The energy metric reveals that the dis- ${ }^{497}$ tributions estimated from CT samples and those from the ${ }^{498}$ KLE model are different at some locations. It has been shown ${ }^{499}$ that five dominant $\mathrm{KL}$ coefficients are sufficient for an accu- ${ }^{500}$ rate reproduction of variance in $\overline{\mathrm{BMD}}$. However, the analysis of the energy distance shows that far more $\mathrm{KL}$ coefficients $(>30)$ are needed to reproduce the distribution function of the BMD random field. Energy distance is stricter than $\overline{\mathrm{BMD}}_{502}$ because it directly describes the similarity of BMD distribu-503 tions. Hence, the energy distance could be a good indicator ${ }_{504}$

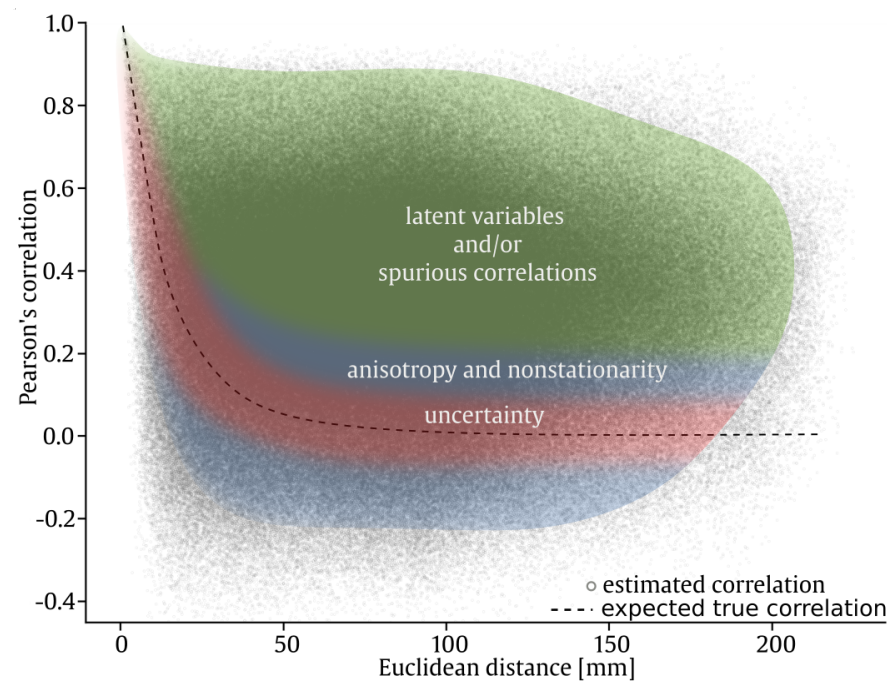

Figure 7: Correlation dependence on distance for a BMD random field for females estimated from CT samples.

that local properties such as stress and deformation quantities might not be accurate enough and mean/std estimation might be biased. To improve our model, the identification of (generally non-Gaussian) distributions of $\mathrm{KL}$ coefficients should be incorporated into a random field model based on $\mathrm{KLE}$, for example by the iterative algorithm introduced in [75].

\section{Random Field Model Implementation}

The covariance matrix of BMD is dense and large, hence it disallows a common storage representation or the solution of a Fredholm integral equation. Although we partially avoided these difficulties by directly manipulating the data on a discrete level, a more robust approach must be applied, for instance the recent approximation of $\mathrm{KL}$ by an isogeometric method [76].

\section{Comparison with Statistical Shape \& Appearance Models (SSM/SSA)}

Our method shares the steps of geometry aligment and spectral decomposition of the empirical covariance matrix with SSM/SSA [77, 78, 79], but the meaning and computing of these steps is different. The bone shape aligment is computed on an ROI of whole pelvic bone, allowing the interior to be aligned as well. Our approach uses covariance eigenpairs as bases for generating new BMD realizations. Most importantly, our approach is rather focused on exploring/explaining the spatio-temporal correlation structure, which somehow reflects the (mechano-)biological mechanisms of growth and adaptation [80] in the authors' opinion.

\section{Conclusion}

The understanding of uncertainties in bone density is of paramount importance to biomechanics in the relation to the understanding of bone mechanobiology, and it should 
bioRxiv preprint doi: https://doi.org/10.1101/2021.02.25.432881; this version posted August 17, 2021. The copyright holder for this preprint (which was not certified by peer review) is the author/funder, who has granted bioRxiv a license to display the preprint in perpetuity. It is made available under aCC-BY-NC-ND 4.0 International license.

be properly incorporated into computational models. We in-559 troduced a random field model describing the fluctuation in ${ }^{560}$ bone density via the KLE. The following sub-conclusions $\operatorname{can}_{562}^{561}$ be drawn:

- BMD has a complex correlation structure which cannot ${ }_{565}^{564}$ be modeled by an isotropic, spatially/temporally sta-566 tionary Gaussian random field,

- Gaussian KL coefficients allow $\overline{\mathrm{BMD}}$ to be simulated ac- ${ }^{569}$ curately, of BMD to be incorporated.

\section{Conflict of interest statement}

The authors declare no conflict of interest.

\section{Acknowledgements}

The authors acknowledge financial support from project ${ }^{586}$ No. LTAUSA19058 provided by the Ministry of Education, ${ }_{588}^{587}$ Youth and Sports of the Czech Republic. Additionally, the ${ }_{589}$ work has been supported by the Czech Science Foundation590 under project No. 20-01781S.

\section{References}

[1] N. Reznikov, R. Shahar, S. Weiner, Bone hierarchical structure in three ${ }_{597}^{596}$ dimensions, Acta Biomaterialia 10 (2014) 3815-3826. doi:10.1016/598 j.actbio.2014.05.024, biomineralization.

[2] B. Busse, M. Hahn, M. Soltau, J. Zustin, K. Püschel, G. N. Duda, M. Am- ${ }^{599}$ ling, Increased calcium content and inhomogeneity of mineralization ${ }_{601}$ render bone toughness in osteoporosis: mineralization, morphology $\mathrm{y}_{602}$ and biomechanics of human single trabeculae, Bone 45 (2009) 10341043. doi:10.1016/j . bone.2009.08.002.

[3] K. Tai, M. Dao, S. Suresh, A. Palazoglu, C. Ortiz, Nanoscale het- ${ }^{604}$ erogeneity promotes energy dissipation in bone, Nature Materials $6_{606}^{605}$ (2007) 454-462. doi:10.1038/nmat1911.

[4] B. Davies, A. King, P. Newman, A. Minett, C. R. Dunstan, H. Zreiqat, ${ }_{60}$ Hypothesis: Bones toughness arises from the suppression of elastic ${ }_{609}$ waves, Scientific reports 4 (2014) 7538. doi:10.1038/srep07538. ${ }_{610}$

[5] A. M. Torres, J. B. Matheny, T. M. Keaveny, D. Taylor, C. M. Rimnac, ${ }_{611}$ C. J. Hernandez, Material heterogeneity in cancellous bone promotes deformation recovery after mechanical failure, Proceedings of the $\mathrm{Na}^{-}{ }_{613}$ tional Academy of Sciences 113 (2016) 2892-2897. doi:10.1073/614 pnas. 1520539113.

[6] D. Ruffoni, P. Fratzl, P. Roschger, K. Klaushofer, R. Weinkamer, The ${ }_{616}^{615}$ bone mineralization density distribution as a fingerprint of the min- ${ }_{617}$ eralization process, Bone 40 (2007) 1308-1319. doi:10.1016/j ${ }_{618}$ bone.2007.01.012.

[7] C. Falcinelli, E. Schileo, L. Balistreri, F. Baruffaldi, B. Bordini, M. Vice- ${ }_{620}^{619}$ conti, U. Albisinni, F. Ceccarelli, L. Milandri, A. Toni, F. Taddei, Mul- ${ }_{621}$ tiple loading conditions analysis can improve the association between ${ }_{622}$ finite element bone strength estimates and proximal femur fractures: ${ }_{623}$ A preliminary study in elderly women, Bone 67 (2014) 71-80. ${ }_{624}$ doi:10.1016/j.bone.2014.06.038.

[8] E. S. Orwoll, L. M. Marshall, C. M. Nielson, S. R. Cummings, J. Lapidus ${ }^{62}$ J. A. Cauley, K. Ensrud, N. Lane, P. R. Hoffmann, D. L. Kopperdahl, ${ }_{627}$ et al., Finite element analysis of the proximal femur and hip fracture risk in older men, Journal of bone and mineral research 24 (2009) ${ }_{629}$ 475-483. doi:10.1359/jbmr. 081201.
[9] G. Dahan, N. Trabelsi, O. Safran, Z. Yosibash, Finite element analyses for predicting anatomical neck fractures in the proximal humerus, Clinical Biomechanics 68 (2019) 114-121. doi:10.1016/ j.clinbiomech.2019.05.028.

[10] X. N. Dong, R. Pinninti, T. Lowe, P. Cussen, J. E. Ballard, D. Di Paolo, M. Shirvaikar, Random field assessment of inhomogeneous bone mineral density from DXA scans can enhance the differentiation between postmenopausal women with and without hip fractures, Journal of Biomechanics 48 (2015) 1043-1051. doi:10.1016/j.jbiomech. 2015.01 .030$.

[11] X. N. Dong, M. Shirvaikar, X. Wang, Biomechanical properties and microarchitecture parameters of trabecular bone are correlated with stochastic measures of 2D projection images, Bone 56 (2013) 327336. doi: $10.1016 / j$. bone 2013.05 .023 .

[12] X. N. Dong, R. Pinninti, A. Tvinnereim, T. Lowe, D. Di Paolo, M. Shirvaikar, Stochastic predictors from the DXA scans of human lumbar vertebrae are correlated with the microarchitecture parameters of trabecular bone, Journal of Biomechanics 48 (2015) 2968-2975. doi:10.1016/j.jbiomech.2015.07.041.

[13] X. N. Dong, Y. Lu, M. Krause, G. Huber, Y. Chevalier, H. Leng, G. Maquer, Variogram-based evaluations of DXA correlate with vertebral strength, but do not enhance the prediction compared to aBMD alone, Journal of Biomechanics 77 (2018) 223-227. doi:10.1016/ j.jbiomech.2018.07.009.

[14] G. Maquer, Y. Lu, E. Dall'Ara, Y. Chevalier, M. Krause, L. Yang, R. Eastell, K. Lippuner, P. K. Zysset, The initial slope of the variogram, foundation of the trabecular bone score, is not or is poorly associated with vertebral strength, Journal of bone and mineral research 31 (2016) 341-346. doi:10.1002/jbmr. 2610.

[15] X. N. Dong, Q. Luo, D. M. Sparkman, H. R. Millwater, X. Wang, Random field assessment of nanoscopic inhomogeneity of bone, Bone 47 (2010) 1080-1084. doi:10.1016/j . bone.2010.08.021.

[16] C. Desceliers, C. Soize, S. Naili, G. Haïat, Probabilistic model of the human cortical bone with mechanical alterations in ultrasonic range, Mechanical Systems and Signal Processing 32 (2012) 170-177. doi:10.1016/j . ymssp. 2012.03.008.

[17] M. Kirby, A. H. Morshed, J. Gomez, P. Xiao, Y. Hu, X. E. Guo, X. Wang, Three-dimensional rendering of trabecular bone microarchitecture using a probabilistic approach, Biomechanics and Modeling in Mechanobiology (2020) 1-19. doi:10.1007/ s10237-020-01286-8.

[18] J. Luque, I. Papaioannou, D. Straub, M. Ruess, D. Schillinger, Probabilistic model of bone structure based on CT scan data and validation of simulation results using the finite cell method, in: ICOSSAR 2012, University of N.Y.C., New York City, 2013, pp. 2017-2024.

[19] J. G. Eastman, J. C. Krieg, M. L. C. Routt Jr, Early failure of symphysis pubis plating, Injury 47 (2016) 1707-1712.

[20] B. R. Moed, C. S. Grimshaw, D. N. Segina, Failure of locked designspecific plate fixation of the pubic symphysis: a report of six cases, Journal of orthopaedic trauma 26 (2012) e71-e75.

[21] S. Andrich, B. Haastert, E. Neuhaus, K. Neidert, W. Arend, C. Ohmann, J. Grebe, A. Vogt, P. Jungbluth, S. Thelen, et al., Excess mortality after pelvic fractures among older people, Journal of Bone and Mineral Research 32 (2017) 1789-1801.

[22] K. Strømsøe, W. Kok, A. Høiseth, A. Alho, Holding power of the 4.5 $\mathrm{mm}$ ao/asif cortex screw in cortical bone in relation to bone mineral, Injury 24 (1993) 656-659.

[23] J. Seebeck, J. Goldhahn, H. Städele, P. Messmer, M. M. Morlock, E. Schneider, Effect of cortical thickness and cancellous bone density on the holding strength of internal fixator screws, Journal of orthopaedic research 22 (2004) 1237-1242.

[24] K. Matsukawa, Y. Yato, R. A. Hynes, H. Imabayashi, N. Hosogane, Y. Yoshihara, T. Asazuma, K. Nemoto, Comparison of pedicle screw fixation strength among different transpedicular trajectories, Clinical spine surgery 30 (2017) 301-307.

[25] G. J. Székely, M. L. Rizzo, Energy statistics: A class of statistics based on distances, Journal of Statistical Planning and Inference 143 (2013) 1249-1272. doi:10.1016/j.jspi.2013.03.018.

[26] Y. Pauchard, T. Fitze, D. Browarnik, A. Eskandari, I. Pauchard, W. EnnsBray, H. Pálsson, S. Sigurdsson, S. J. Ferguson, T. B. Harris, et al., Interactive graph-cut segmentation for fast creation of finite element mod- 
bioRxiv preprint doi: https://doi.org/10.1101/2021.02.25.432881; this version posted August 17, 2021. The copyright holder for this preprint (which was not certified by peer review) is the author/funder, who has granted bioRxiv a license to display the preprint in perpetuity. It is made available under aCC-BY-NC-ND 4.0 International license.

els from clinical CT data for hip fracture prediction, Computer meth-70 ods in biomechanics and biomedical engineering 19 (2016) 1693-702 1703. doi:10.1080/10255842.2016.1181173.

[27] A. S. Michalski, B. A. Besler, G. J. Michalak, S. K. Boyd, CT-based704 internal density calibration for opportunistic skeletal assessment using705 abdominal CT scans, Medical Engineering \& Physics 78 (2020) 55-63.706 doi:10.1016/j.medengphy .2020.01.009.

[28] M. Kuchař, P. Henyš, P. Rejtar, P. Hájek, Shape morphing technique708 can accurately predict pelvic bone landmarks, International Journal709 of Legal Medicine (2021). doi:10.1007/s00414-021-02501-6. 710

[29] B. B. Avants, N. J. Tustison, G. Song, P. A. Cook, A. Klein, J. C.711 Gee, A reproducible evaluation of ANTs similarity metric per-712 formance in brain image registration, Neuroimage 54 (2011)713 2033-2044. doi:10.1016/j.neuroimage.2010.09.025, pMID:714 20851191 PMCID: PMC3065962.

[30] B. B. Avants, P. Yushkevich, J. Pluta, D. Minkoff, M. Korczykowski,716 J. Detre, J. C. Gee, The optimal template effect in hippocampus717 studies of diseased populations, Neuroimage 49 (2010) 2457-2466.718 doi:10.1016/j.neuroimage.2009.09.062.

31] B. L. Will Schroeder, Ken Martin, The visualization toolkit: an object-720 oriented approach to 3D graphics, Kitware, 2003.

[32] Y. Hu, T. Schneider, B. Wang, D. Zorin, D. Panozzo, Fast tetrahe- 722 dral meshing in the wild, arXiv (2019) arXiv-1908. doi:10.1145/723 3386569.3392385.

[33] M. Vořechovský, Simulation of simply cross correlated random fields725 by series expansion methods, Structural safety 30 (2008) 337-363.726 doi:10.1016/j.strusafe.2007.05.002.

[34] H. Harbrecht, M. Peters, M. Siebenmorgen, Efficient approximation of 72 random fields for numerical applications, Numerical Linear Algebra729 with Applications 22 (2015) 596-617. doi:10.1002/nla.1976. 730

[35] R. G. Ghanem, P. D. Spanos, Stochastic finite elements: a73 spectral approach, Courier Corporation, 2003. doi:10.1007/732 978-1-4612-3094-6.

[36] W. Conover, On a better method for selecting input variables, 734 1975. URL: https://prod-ng.sandia.gov/techlib-noauth/735 access-control.cgi/2001/010417.pdf, unpublished Los736 Alamos National Laboratories manuscript, reproduced as Appendix A737 of "Latin Hypercube Sampling and the Propagation of Uncertainty in738 Analyses of Complex Systems" by J.C. Helton and F.J. Davis, Sandia739 National Laboratories report SAND2001-0417, printed November740 2002.

[37] M. D. McKay, W. J. Conover, R. J. Beckman, A comparison of three 74 methods for selecting values of input variables in the analysis of output743 from a computer code, Technometrics 21 (1979) 239-245. doi:10.744 1080/00401706.1979.10489755.

[38] Petr Henyš et al., BoneGen, http://bonegen.nti.tul.cz/746 BoneGen_server_version, 2021.

[39] M. A. Juárez, M. Pennisi, G. Russo, D. Kiagias, C. Curreli, M. Vice-748 conti, F. Pappalardo, Generation of digital patients for the simulation 749 of tuberculosis with UISS-TB, BMC Bioinformatics 21 (2020) 1-8.750 doi:10.1186/s12859-020-03776-z.

[40] M. Viceconti, F. Pappalardo, B. Rodriguez, M. Horner, J. Bischoff, F. M.752 Tshinanu, In silico trials: Verification, validation and uncertainty753 quantification of predictive models used in the regulatory evaluation 754 of biomedical products, Methods (2020). doi:10.1016/j.ymeth.755 2020.01 .011$.

[41] T. M. Keaveny, D. L. Kopperdahl, L. J. Melton III, P. F. Hoffmann,757 S. Amin, B. L. Riggs, S. Khosla, Age-dependence of femoral strength 758 in white women and men, Journal of bone and mineral research 25759 (2010) 994-1001. doi:10.1359/jbmr.091033.

[42] F. Yu, Y. Xu, Y. Hou, Y. Lin, R. Jiajue, Y. Jiang, O. Wang, M. Li,761 X. Xing, L. Zhang, L. Qin, E. Hsieh, W. Xia, Age-, site-, and sex-762 specific normative centile curves for HR-pQCT -derived microarchi-763 tectural and bone strength parameters in a chinese mainland popula-764 tion, Journal of Bone and Mineral Research 35 (2020) 2159-2170.765 doi:10.1002/jbmr. 4116.

[43] J. Sanchez-Siles, I. Tamimi-Mariño, A. Cortes, J. Ackerman,767 D. González-Quevedo, E. Guerado, A. García, F. Yaghoubi, M. Abdal-768 lah, H. Eimar, et al., Age related changes in the bone microstructure769 in patients with femoral neck fractures, Injury (2020). doi:10.1016/770 j. injury.2020.02.014.
[44] D. M. Patton, E. M. Bigelow, S. H. Schlecht, D. H. Kohn, T. L. Bredbenner, K. J. Jepsen, The relationship between whole bone stiffness and strength is age and sex dependent, Journal of Biomechanics 83 (2019) 125-133. doi:10.1016/j.jbiomech.2018.11.030.

[45] T. Gross, D. H. Pahr, P. K. Zysset, Morphology-elasticity relationships using decreasing fabric information of human trabecular bone from three major anatomical locations, Biomechanics and modeling in mechanobiology 12 (2013) 793-800. doi:10.1007/ s10237-012-0443-2.

[46] P. Varga, P. Zysset, Assessment of volume fraction and fabric in the distal radius using HR-pQCT, Bone 45 (2009) 909-917. doi:10.1016/ j.bone.2009.07.001.

[47] D. Larsson, B. Luisier, M. E. Kersh, E. Dall'Ara, P. K. Zysset, M. G. Pandy, D. H. Pahr, Assessment of transverse isotropy in clinical-level CT images of trabecular bone using the gradient structure tensor, Annals of biomedical engineering 42 (2014) 950-959. doi:10.1007/ s10439-014-0983-y.

[48] G. Dougherty, D. Newman, Measurement of thickness and density of thin structures by computed tomography: a simulation study, Medical Physics 26 (1999) 1341-1348. doi:10.1118/1.598629.

[49] S. Prevrhal, J. C. Fox, J. A. Shepherd, H. K. Genant, Accuracy of CTbased thickness measurement of thin structures: Modeling of limited spatial resolution in all three dimensions, Medical Physics 30 (2003) 1-8. doi:10.1118/1.1521940.

[50] J. Hernigou, A. Alves, Y. Homma, I. Guissou, P. Hernigou, Anatomy of the ilium for bone marrow aspiration: map of sectors and implication for safe trocar placement, International Orthopaedics 38 (2014) 2585-2590. doi:10.1007/s00264-014-2353-7.

[51] G. Treece, A. Gee, Independent measurement of femoral cortical thickness and cortical bone density using clinical CT, Medical Image Analysis 20 (2015) 249-264. doi:10.1016/j.media.2014.11.012.

[52] D. C. Lee, P. F. Hoffmann, D. L. Kopperdahl, T. M. Keaveny, Phantomless calibration of ct scans for measurement of bmd and bone strength-inter-operator reanalysis precision, Bone 103 (2017) 325333. doi:https://doi.org/10.1016/j.bone.2017.07.029.

[53] C. Winsor, X. Li, M. Qasim, C. Henak, P. Pickhardt, H. Ploeg, M. Viceconti, Evaluation of patient tissue selection methods for deriving equivalent density calibration for femoral bone quantitative CT analyses, Bone 143 (2021) 115759. doi:10.1016/j.bone.2020. 115759.

[54] O. Demontiero, C. Vidal, G. Duque, Aging and bone loss: new insights for the clinician, Therapeutic advances in musculoskeletal disease 4 (2012) 61-76. doi:10.1177/1759720X11430858.

[55] S. Khosla, B. L. Riggs, Pathophysiology of age-related bone loss and osteoporosis, Endocrinology and Metabolism Clinics 34 (2005) 10151030. doi:10.1016/j.ecl.2005.07.009.

[56] B. L. Riggs, L. J. Melton III, R. A. Robb, J. J. Camp, E. J. Atkinson, J. M. Peterson, P. A. Rouleau, C. H. McCollough, M. L. Bouxsein, S. Khosla, Population-based study of age and sex differences in bone volumetric density, size, geometry, and structure at different skeletal sites, Journal of Bone and Mineral Research 19 (2004) 1945-1954. doi:10.1359/ JBMR. 040916.

[57] X.-H. Ma, W. Zhang, Y. Wang, P. Xue, Y.-K. Li, Comparison of the spine and hip BDM assessments derived from quantitative computed tomography, International Journal of Endocrinology 2015 (2015). doi:10.1155/2015/675340.

[58] E. G. Vajda, R. D. Bloebaum, Age-related hypermineralization in the female proximal human femur, The Anatomical Record 255 (1999) 202211. doi:10.1002/(sici) 1097-0185(19990601) 255:2<202: : aid-ar10>3.0.co;2-0.

[59] A. Paschall, A. H. Ross, Biological sex variation in bone mineral density in the cranium and femur, Science \& Justice 58 (2018) 287-291. doi:10.1016/j.scijus.2018.01.002.

[60] S. M. Ott, Cortical or trabecular bone: what's the difference?, American journal of nephrology 47 (2018) 373-376. doi:10.1159/ 000489672 .

[61] X. Wang, Cortical bone mechanics and composition: effects of age and gender, in: Skeletal Aging and Osteoporosis, Springer, 2012, pp. 53-85. doi:10.1007/8415_2011_108.

[62] E. Seeman, Structural basis of growth-related gain and age-related loss of bone strength, Rheumatology 47 (2008) iv2-iv8. doi:10.1093/ 
rheumatology/ken177, proceedings of a satellite symposium held 843 on the occasion of the EULAR Congress, Paris, France, June 13, 2008.844

[63] E. F. Kranioti, A. Bonicelli, J. G. García-Donas, Bone-mineral density:845 clinical significance, methods of quantification and forensic applica-846 tions, Research and Reports in Forensic Medical Science 9 (2019) 9-21. doi:10.2147/RRFMS . S164933.

[64] A. Ural, D. Vashishth, Hierarchical perspective of bone toughness-from molecules to fracture, International Materials Reviews 59 (2014) 245263. doi:10.1179/1743280414Y.0000000031.

[65] K. J. Jepsen, N. Andarawis-Puri, The amount of periosteal apposition required to maintain bone strength during aging depends on adult bone morphology and tissue-modulus degradation rate, Journal of Bone and Mineral Research 27 (2012) 1916-1926. doi:10.1002/ jbmr. 1643 .

[66] K. M. Nicks, S. Amin, E. J. Atkinson, B. L. Riggs, L. J. Melton III, S. Khosla, Relationship of age to bone microstructure independent of areal bone mineral density, Journal of Bone and Mineral Research 27 (2012) 637-644. doi:10.1002/ jbmr. 1468.

[67] J. E. Aaron, N. B. Makins, K. Sagreiya, The microanatomy of trabecular bone loss in normal aging men and women, Clinical Orthopaedics and Related Research 215 (1987) 260-271. doi:10 . 1097/ 00003086-198702000-00038, pMID: 3802645.

[68] M. Ding, A. Odgaard, F. Linde, I. Hvid, Age-related variations in the microstructure of human tibial cancellous bone, Journal of Orthopaedic Research 20 (2002) 615-621. doi:10.1016/ S0736-0266 (01) 00132-2.

[69] K. J. Jepsen, Functional interactions among morphologic and tissue quality traits define bone quality, Clinical Orthopaedics and Related Research ${ }^{\circledR} 469$ (2011) 2150-2159. doi:10.1007/ s11999-010-1706-9.

[70] H. Chen, X. Zhou, H. Fujita, M. Onozuka, K.-Y. Kubo, Age-related changes in trabecular and cortical bone microstructure, International journal of endocrinology 2013 (2013). doi:10 .1155/2013/213234.

[71] T. Koehne, E. Vettorazzi, N. Küsters, R. Lüneburg, B. Kahl-Nieke, K. Püschel, M. Amling, B. Busse, Trends in trabecular architecture and bone mineral density distribution in 152 individuals aged 30-90 years, Bone 66 (2014) 31-38. doi:10.1016/j . bone. 2014.05.010.

[72] S. Pezzuto, A. Quaglino, M. Potse, On sampling spatially-correlated random fields for complex geometries, in: International Conference on Functional Imaging and Modeling of the Heart, Springer, 2019, pp. 103-111. doi:10.1007/978-3-030-21949-9_12.

[73] V. S. Cheong, B. C. Roberts, V. Kadirkamanathan, E. Dall'Ara, Bone remodelling in the mouse tibia is spatio-temporally modulated by oestrogen deficiency and external mechanical loading: A combined in vivo/in silico study, Acta Biomaterialia 116 (2020) 302-317. doi:10 . 1016/j.actbio.2020.09.011.

[74] J. Bun, J.-P. Bouchaud, M. Potters, Cleaning large correlation matrices: Tools from random matrix theory, Physics Reports 666 (2017) 1109. doi:10.1016/j.physrep.2016.10.005, cleaning large correlation matrices: tools from random matrix theory.

[75] Z. Zheng, H. Dai, Y. Wang, W. Wang, A sample-based iterative scheme for simulating non-stationary non-Gaussian stochastic processes, Mechanical Systems and Signal Processing 151 (2021) 107420. doi:10.1016/j.ymssp. 2020.107420.

[76] M. Ł. Mika, T. J. R. Hughes, D. Schillinger, P. Wriggers, R. R. Hiemstra, A matrix-free isogeometric Galerkin method for Karhunen-Loève approximation of random fields using tensor product splines, tensor contraction and interpolation based quadrature, arXiv preprint arXiv:2011.13861 (2020). URL: https : //arxiv . org/abs/2011. $13861 \mathrm{v} 1$.

[77] N. Sarkalkan, H. Weinans, A. A. Zadpoor, Statistical shape and appearance models of bones, Bone 60 (2014) 129-140. doi:10.1016/ j.bone.2013.12.006.

[78] V. Chandran, G. Maquer, T. Gerig, P. Zysset, M. Reyes, Supervised learning for bone shape and cortical thickness estimation from CT images for finite element analysis, Medical Image Analysis 52 (2019) 42-55. doi:10.1016/j.media.2018.11.001.

[79] S. P. Väänänen, L. Grassi, G. Flivik, J. S. Jurvelin, H. Isaksson, Generation of 3D shape, density, cortical thickness and finite element mesh of proximal femur from a DXA image, Medical Image Analysis 24 (2015) 125-134. doi:10.1016/j.media.2015.06.001.
[80] T. J. Paul, P. Kollmannsberger, Biological network growth in complex environments: A computational framework, PLOS Computational Biology 16 (2020) e1008003. doi:10.1371/journal.pcbi. 1008003. 